\title{
Language and Cognitive Processes
}

\section{Changes in frame of reference use across the preschool years: A longitudinal study of the gestures and speech produced during route descriptions}

\section{Kazuki Sekine}

To cite this article: Kazuki Sekine (2009) Changes in frame of reference use across the preschool years: A longitudinal study of the gestures and speech produced during route descriptions, Language and Cognitive Processes, 24:2, 218-238, DOI: 10.1080/01690960801941327

To link to this article: http://dx.doi.org/10.1080/01690960801941327

曲 Published online: 23 Jan 2009.

Submit your article to this journal $₫$

Џll Article views: 148

View related articles ־

Citing articles: 4 View citing articles 


\title{
Changes in frame of reference use across the preschool years: A longitudinal study of the gestures and speech produced during route descriptions
}

\author{
Kazuki Sekine \\ Shirayuri College, Tokyo, Japan
}

This study longitudinally investigated developmental changes in the frame of reference used by children in their gestures and speech. Fifteen children, between 4 and 6 years of age, were asked once a year to describe their route home from their nursery school. When the children were 4 years old, they tended to produce gestures that directly and continuously indicated their actual route in a large gesture space. In contrast, as 6-year-olds, their gestures were segmented and did not match the actual route. Instead, at age 6 , the children seemed to create a virtual space in front of themselves to symbolically describe their route. These results indicate that the use of frames of reference develops across the preschool years, shifting from an actual environmental to an abstract environmental frame of reference. Factors underlying the development of frame of reference, including verbal encoding skills and experience, are discussed.

Information about a route is important not only for adults, but also for children in their everyday life. In travelling between their home and their nursery school or kindergarten nearly every day, how do preschoolers understand their environment? In the present study, I examined the frameworks that children use to recall the route from their school to their own home, and how their understanding of the environment is expressed in their route description. I took a longitudinal approach to investigate the

Correspondence should be addressed to Kazuki Sekine, Department of Developmental Psychology, Shirayuri College, 1-25 Midorigaoka Chofu-shi, Tokyo 182-8525, Japan. E-mail: kazuki@tkc.att.ne.jp

I would like to thank Suzuki Tadashi and Bryan Ho for insightful comments on earlier versions of this manuscript. I would also like to thank the children, parents, and teachers for their cooperation.

C 2008 Psychology Press, an imprint of the Taylor \& Francis Group, an Informa business http://www.psypress.com/lcp

DOI: $10.1080 / 01690960801941327$ 
developmental changes in the gestures and speech used in children's route descriptions.

A spatial frame of reference is regarded as a coordinate system or a framework that an individual uses to spatially locate themselves or objects with respect to the environment or other objects. Piaget (Piaget \& Inhelder, 1956; Piaget, Inhelder, \& Szeminska, 1960) was the first to conduct a systematic investigation of children's organisation of large-scale environmental knowledge. Piaget et al. (1960) asked children between 4 and 12 years of age to reconstruct a model or describe a route around their school; from their responses he defined three developmental stages. In the first stage, which extends to around 7 years of age, children describe a route relative to themselves or their own actions. In the second stage, which typically develops between 7 and 9 years of age, children are able to organise their environment based on clusters of locations around landmarks such as intersections, buildings, and bridges. Finally, between 8 to 12 years of age, children enter the third stage and are able to describe a route consistently using fully coordinated landmarks. Based upon Piaget's theoretical framework, Hart and Moore (1973) proposed a systematic developmental model of frames of reference corresponding to Piaget's three stages. The egocentric frame of reference, fixed frame of reference, and coordinated frame of reference correspond to stages one through three, respectively.

Although these developmental models have some utility in assessing developmental stages, unfortunately Piaget et al. (1960) and Hart and Moore (1973) did not show quantifiable data. Given that some research in smallscale environments has shown that preschoolers tend to code location with respect both to their own body position, and also to external landmarks (Craton, Elicker, Plumert, \& Pick, 1990; Newcombe \& Huttenlocher, 1992), it seems that frame of reference may change even at a preschool age, in contrast to Piaget et al.'s (1960) assertion that young children predominantly use an egocentric frame of reference. In addition, as Craton et al. (1990) noted, few empirical studies have focused on the types of reference objects children spontaneously use in their descriptions of locations, especially in large-scale environments. Therefore, further research is needed to examine the frame of reference type that preschoolers use spontaneously in a largescale environment.

To date, the methods used in research on spatial cognition have made it difficult to grasp the spontaneous use of frames of reference. For example, although recognition methods, including maps, aerial photographs, and environmental models, keep stimuli constant, these presentation modes may provide subjects with cues about the frameworks in advance. Production methods, such as verbal descriptions and sketch maps, are also problematic because they are influenced by the skills children possess. Thus, because these methods force participants to transform their spatial expression into 
information on a two-dimensional plane, and require drawing skills and verbal competence, the result may lead us to underestimate children's spatial performance or their spontaneous use of frames of reference.

To overcome the methodological problems described above, the present study primarily focused on spontaneous gestures as an index of frame of reference. Importantly, gestures can be projected in three-dimensional space and producing gestures is easy, even for preschoolers. Although Piaget et al. (1960) noted the importance of gestures in children in the comment, 'the use of gestures and calling a motor schema to mind are far more helpful than true representation' (p. 12), they did not conduct a detailed, empirical investigation into the use of gestures. Similarly, subsequent studies of children's spatial expression have mainly focused on linguistic aspects and paid little attention to gestures (Blades \& Medlicott, 1992; Gauvain \& Rogoff, 1989; Waller, 1986).

Studies focused on gestures have revealed that people communicating spatial information tend to produce more of the spontaneous gestures (hereinafter referred to as a 'gesture') that co-occur with speech (McNeill, 1992) than those communicating non-spatial information (Rauscher, Krauss, \& Chen, 1996). More importantly, gestures are a primary vehicle for conveying spatial information, especially in young children. This was demonstrated in Doherty-Sneddon and Kent's (1996) study, where they found that gestures occupied a higher proportion of preschoolers' spatial expressions when compared with the expressions of 11-year-olds.

In recent years, research focused on the relation between gestures and frames of reference have increased, although most of the studies are on adults (Haviland, 1993; Kita, 1998; Levinson, 2003). Kita (1998) analysed gestures produced by participants describing a route in a university campus, and reported that they used two types of gestures that were different in direction indication, which were not types of linguistic descriptions. He named these geo-anchored gestures and body-anchored gestures. In geoanchored gestures, the direction of the gestures is determined by the actual locations, whereas in body-anchored gestures, the left and right sides of the speaker's body determined the direction of the gestures. It was also found that when people used geo-anchored gestures, the hand was raised higher above the shoulder, and the elbow was extended more fully than when they used body-anchored gestures, in which the vector of gestures did not usually match the target locations. Based on the analysis of gestural indication of direction, Kita argued that people can use at least two frames of reference for route descriptions; one is a frame of reference that directly indicates the target direction with the explanation situation as the reference point, and the other is a frame of reference that indirectly indicates the target direction by using the speaker's body. In the later case origo (the origin of coordination) seems to be transposed on some place other than the explanation situation. 
In this study, the former frame of reference is referred to as an actual environmental frame of reference, and the latter is referred to as an abstract environmental frame of reference that is inferred by both gestures and speech.

Characteristics of geo-anchored gestures are similar to those found in gestures in language communities such as Guugu Yimithirr or Tzeltal, where an absolute frame of reference (e.g., descriptions based on cardinal directions such as north/south or geographical features) is typically used (Levinson, 2003). Levinson (2003) reported that people who use mainly absolute frame of reference in their language tend to use larger space for gestures when describing a large-scale environment than people from language communities such as English and Japanese, who predominantly use a relative frame of reference in the language (e.g., a description based on left/right or back/forth).

Thus, studies indicate that spontaneous gestures can reflect the frame of reference that people use in their descriptions, and the characteristics of gestures vary depending on whether the speaker can rely on the actual external environment. Both the characteristic of children's directional gestures and whether frames of reference change during the preschool years remain to be definitively determined. According to previous studies assessing children's descriptions of routes, as children begin to learn left/right terms and landmarks, they can segment the route by using those terms in their descriptions (Gauvain \& Rogoff, 1989; Waller, 1986). If gestures and speech form a single integrated system and develop in parallel as McNeill (1992) has argued, gestures would be influenced by acquisition of the spatial terms that segment the route. Thus, the relationship between gesture and speech in terms of segmentation of space should be investigated.

In order to infer frame of reference information in preschool children based on their gestures, the present study borrowed Kita's (1998) categorisation of gestural indication of direction described above. This type of gestural indication could be used to determine if the frame of reference depended on reference objects that the children used as cues in their recall. In Piaget et al.'s (1960) classification of frame of reference, they focused largely on whether children could make use of a movable reference point (namely, the origin of coordinates), their body, or a fixed reference point such as a landmark in the environment. With this classification it is not clear whether children directly orient their body to the actual environment to recall the route, although bodily orientation is considered. Because some researchers using small-scale environments have noted that younger children tend to heavily rely on external landmarks (e.g., furniture, doors, windows, etc.) to code locations in their spatial field (Craton et al., 1990; Newcombe \& Huttenlocher, 1992; Presson, 1980; Presson \& Somerville, 1985), it is critical to determine the connection between the children's bodily orientation and the external 
environment as a reference object. Indeed, in a cross-sectional investigation of preschooler's route descriptions, Sekine (2006) found that 4-year-olds oriented their body directly to an actual route more often than did 6-yearolds. However, in Sekine's study the routes described by participants varied with each child. Thus, the possibility that the results were influenced by individual route characteristics cannot be excluded.

To control for differences in individual routes across age groups, the present study took a longitudinal approach in assessing developmental change in frames of reference used in gesture and speech. Each child described the same route three times, once each year from 4 to 6 years of age. There remained some inter-participant route differences, but in this study, these differences in route characteristics are controlled and thus we can investigate how a child changed the frame of reference of the route during a 3 -year period. The chosen task required the children to explain the route from their nursery school to their home in light of ecological validity. To date, there have been few empirical, longitudinal studies concerning the relationship between gesture and speech in young children, especially above 2 years of age (McNeill, 2005). By studying gestures, I attempted to infer the spontaneous use of frames of reference in preschoolers. Thus, the present study was designed to provide insight not only into the developmental changes of large-scale spatial representations, but also into the development of the relationship between gestures and speech in preschoolaged children.

\section{METHOD}

\section{Participants}

Fifteen children ( 8 male and 7 female) were tested once each February for 3 consecutive years. At the time of their first test, the average age was 54 months $S D=4.13$ ), the next test occurred when the children were 66 months on average, and the final test at an average age of 78 months. Based on these averaged ages, the children are hereafter referred to as 4-, 5-, and 6-year-olds. All participants were native Japanese speakers from middle-class families who attended a public nursery school in Tokyo, Japan. ${ }^{1}$

The nursery school was located in a hilly residential area and was surrounded by a well-maintained road network. The children's homes were plotted on a $1 / 15,000$ scale map and their direct distance to the nursery school was calculated. The average direct distances was $671.0 \mathrm{~m}$ (range $=$

\footnotetext{
${ }^{1}$ The author attended this nursery school once a week for six years as a volunteer to support the teacher. Therefore, the author was familiar with the children who participated in this study, the return routes to their homes, as well as their teacher.
} 
365.2 to $1628.8 \mathrm{~m}$ ). Although there were some children who explained the existence of a different route to their home, all children described the same main route in all three sessions. The children's main commute was classified as either by walking, cycling, or car. Four children walked, three rode a bicycle, and eight rode in a car. No relationship was found between route description and distances or type of commute type, and these factors are not subsequently mentioned.

\section{Procedure}

In order to obtain the children's route description from the nursery school to their own home, interviews were conducted individually in a quiet spare room of the nursery school. An armless chair was placed in the room, in the direction of the nursery school gate, and a video camera was positioned at a 45 degree angle from the child. The curtain covering the room's window was closed, disallowing the children to see outside. Before entering the room, the experimenter and the child confirmed the location and direction of the gate from a window in the corridor immediately outside of the room; they then entered the room. The child sat down in the chair and the experimenter sat facing the child. After developing rapport, the experimenter asked the child, 'Can you tell me how to get from the gate of the nursery school to your home?'. If the participant appeared nervous with the setting or did not seem to understand the instruction, after ensuring the child's knowledge about the direction of the nursery school gate, the experimenter asked, 'How do you go back to your home from the gate of nursery school?'. All children interviewed responded to the question by the second prompt. If a child paused during the description, the experimenter facilitated their continuation of the description by saying 'then?', 'and next?', and so forth. The route descriptions produced by children who required the second prompt did not seem to be different from those of children who responded to the first question. All interviews were recorded by a camcorder. The same procedure was repeated during the subsequent two yearly examinations. In second and third year, before the interview began, the experimenter asked the child whether he/she remembered describing their own route in previous year(s), but none of the participants remembered.

\section{Coding speech and gesture}

Speech performance. The children's spoken and gestured responses were transcribed from the videotapes. To investigate speech performance in route descriptions, the mean number of the following indices were calculated: total speaking time (time spent on the description), total morphemes (excluding fillers such as 'uh', 'ah', hesitations, and speech errors), and total pausing time (a silence lasting more than 0.2 seconds was deemed a pause). In 
addition, the number of landmarks (e.g., a park, river, hospital, and so forth, with the two exceptions of the nursery school and child's own home), the number of motion verbs (e.g., go, walk, run, and turn) and the number of left। right terms were also counted.

Gesture performance. In order to investigate gesture performances in route descriptions, the total number of gestures and the frequency of gestures per second were calculated. Following Iverson's criterion (1999), hand movements were classified as gestures only when they had an identifiable beginning and a clear end, and they were synchronised with speech. Due to their infrequent occurrence, the repetitive up and down hand movements known as beats were excluded from present study. One 5-year-old girl in this study did not produce any gestures at all. Although her data were included in the analysis of the total number and frequency of gestures, she was excluded from later analyses examining the proportions of gestures and a correlation analysis.

Direction of gestures. To examine whether the children oriented themselves to the target direction or location, children were classified into three groups based on their consistency/inconsistency between the direction of their gestures and the direction of the actual route. When children consistently produced gestures that matched their actual route home throughout the description, they were categorised within the match group. Following Kita (1998), gestures were considered a match, if they fell within \pm 45 degrees of the target vector. Due to the recording conditions, a more precise criterion was not possible. In contrast, when children consistently produced gestures that did not match with their actual route, they were categorised within the mismatch group. Typically, children in the mismatch group produced gestures as if making a virtual space in front of themselves, without regard for orientation to the actual environment. Children who used both match and mismatch gestures in their descriptions were categorised as the mixed group. In the mixed group, a child might explain the first turn in the route, while gesturing in the actual direction of the turn, but subsequently their gestures would not match the target location or direction.

Gesture space. To investigate the use of space in route descriptions, the proportion of gesture space used was examined. Gesture space was defined as the space assumed in front of a speaker where gestures are produced when the speaker is seated (McNeill, 1992). The gesture space may be divided into the following four sectors using a system of concentric squares: CentreCentre (in front of the chest), Centre (surrounding the trunk of the body), Periphery (surrounding from the head to the knee), and Extreme Periphery (the most external sector). The production rate of gestures in each sector was 
calculated. In this study, because the children often produced gestures while twisting at their waist or turning away, a median line dividing their trunk vertically was treated as the central axis of gesture space.

Gesture morphology. Kita (1998) found that people used geo-anchored gestures that directly indicated the actual route. Specifically, a geoanchored gesture is more likely to be performed with fully extended elbow and raised hand above the shoulder level compared with a body-anchored gesture. To investigate whether the same tendencies could be seen in the present study, criteria established in previous studies (Kita, 1998; Levinson, 2003) were adopted; the number of gestures with a fully extended elbow and the number of gestures with the entire hand from the wrist up raised above shoulder level at some point during the stroke phase were calculated. Each measure was divided by the total number of gestures, respectively, and the mean proportions were calculated for each age.

Gesture unit. To examine segmentation of the route by gesture, the total number of up and down movements of the arm, called gesture units, was counted. A gesture unit is defined as the period between successive rests of the limb (Kendon, 1980). A gesture unit begins the moment the limb begins to move and ends when it rests back at the original position (McNeill, 1992). A gesture unit often includes multiple gestures that consist of one or more movement phases. Gestures may be maintained in the air during a description, or may involve preparation for the next gesture. The mean of the total number of gesture units was calculated.

Reliability. For each route description, an author and a trained graduate student independently judged the number of target behaviours for each speech and gestural index. When the two coders gave exactly the same number (e.g., the number of morphemes) for a given route description, it counted as agreement, and when the number differed (e.g., 8 vs. 7) then it counted as disagreement. Agreements between two coders were calculated for each index. The two coders agreed in more than $94 \%$ of the route descriptions for all speech indices including the number of morphemes, landmarks, motion verbs, and left/right terms, and in more than $85 \%$ of the route descriptions for all gestural indices including the number of gestures, gestures that had fallen into each gesture space, gestures with a fully extended elbow and with a hand raised above the shoulder, and gesture unit. Reliability of the direction of gestures was assessed independently by an experimenter and a nursery teacher trained in coding gestures who knew the children's routes home. The two coders agreed on the direction coding in $87 \%$ of the gestures. Any coding disagreements were resolved through discussion. 


\section{RESULTS}

Analyses were conducted as follows. First, the expression of turns was investigated. Next, general age differences in route descriptions were investigated by analysing speech and gesture performance. Finally, the spatial characteristics of gestures, such as the direction, use of space, gesture morphology, and gesture units, were investigated. From the characteristics found in verbal descriptions and gestures, the frame of reference used by each age group was inferred.

\section{Linguistic and gesture expressions of a turn}

To travel from the school to their own home, each child had to navigate several turns. The average number of turns on the way home from the nursery school was 6.9 (range $=4-9)$. The quantity and type by which turns were expressed in gesture and speech were investigated by calculating the number of turns and the type of expression. All turns were classified into one of four expression types: (a) expression in both gesture and speech, (b) no expression at all, (c) expression in only speech, and (d) expression in only gesture. No expression at all indicated that the child failed to mention a turn that they should have mentioned. The total number of each expression type in all turns was calculated for each age, as shown in Figure 1.

Chi-square analysis was used to determine the relationship between expression style and age, and a significant relationship was found, $\chi^{2}(6, n=$

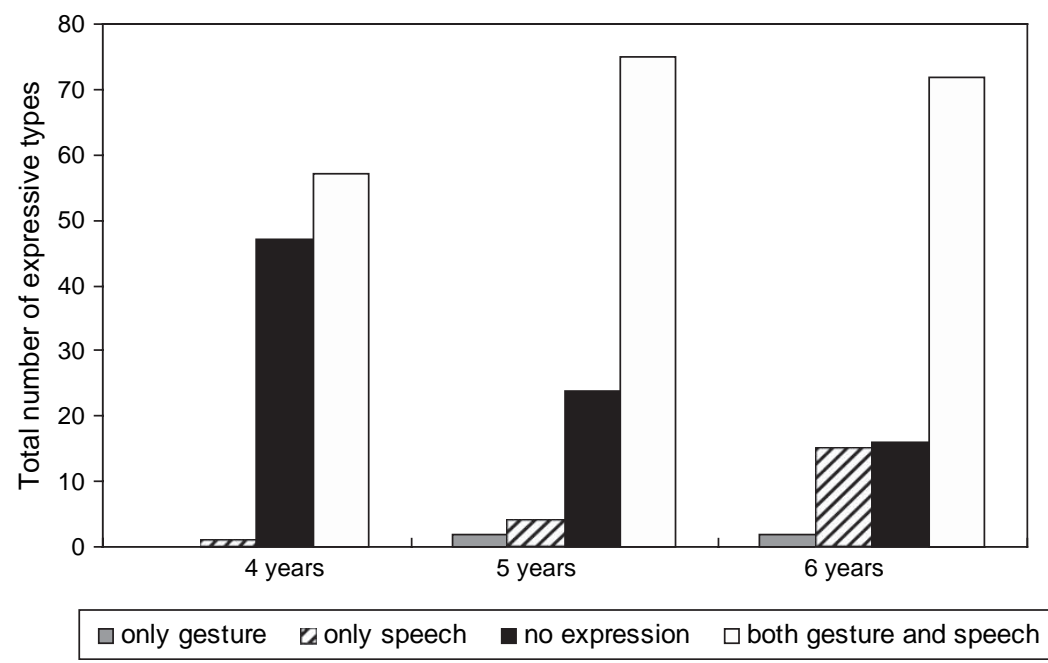

Figure 1. The total number of turn expression types. The figure shows the total number of four expression types in all turns at each age. 
$315)=38.9, p<.001$. A residual analysis indicated that in 4-year-olds, although there were significantly fewer turns expressed by 'both gesture and speech' and by 'only speech', there were significantly more turns that had 'no expression at all'. In contrast, 6-year-olds mentioned significantly more turns by 'only speech', and there were fewer turns that had 'no expression at all'. In order to test if children changed their expression style over the 3-year period, Cochran's $Q$ test was carried out for each expression type, with McNemar tests for post hoc pair-wise comparisons. First, each child in a given session was classified according to whether or not $\mathrm{s} / \mathrm{he}$ used 'only speech' at least once to express the turns in the route. The number of children who used 'only speech' significantly changed over the 3 years, $Q(2$, $15)=7.143, p<.05$. Pairwise comparisons indicated that there was a marginal significance between 6-year-olds and 5-year-olds (two-tailed McNemar test, $p=.063$ ). There were no statistically significant changes over the 3 years for the other expression types.

It appears that, as children get older, they can express most of the turns on their way home by using both gesture and speech together, especially 6-yearolds, in comparison to 5-year-olds, more often expressed a turn using only speech, and they failed to mention turns less frequently than they did when they were younger.

\section{Speech performances}

A repeated-measures analysis of variance (ANOVA) was conducted on speech performance, with age as a within-subjects factor (Table 1). Results revealed a main effect of age on speaking time, $F(2,28)=12.87, p<.01$, total morphemes, $F(2,28)=9.69, p<.001$, pausing time, $F(2,28)=7.04, p<.01$, motion verb use, $F(2,28)=9.39, p<.01$, left/right term use, $F(2,28)=7.20$, $p<.05$, and landmark use, $F(2,28)=9.48, p<.01$. Post hoc comparisons (Bonferroni, $p<.05$ ) indicated that the average scores for speech indices of 6 -year-olds were higher than those of 4-year-olds across all of the speech indices. For speaking time, the average for the 6-year-olds exceeded that of the 5-year-olds, who in turn had higher scores in speaking time and motion verb use than the 4-year-olds.

Next, the number of children who mentioned left/right terms and landmarks more than once during their descriptions was investigated. Use of left/right terms was $0(0 \%)$ for 4 -year-olds, 4 (27\%) for 5-year-olds, and 7 $(47 \%)$ for 6 -year-olds. Similarly, the number of children who referred to landmarks was $8(53 \%)$ for 4-year-olds, $13(87 \%)$ for 5-year-olds, and 15 $(100 \%)$ for 6-year-olds. Cochran's $Q$ test was used to analyse this data. It demonstrated significant relationships between age and use of left/right terms and landmarks, $Q(2,15)=9.25, p<.01 ; Q(2,15)=9.75, p<.01$, respectively. McNemar tests for post hoc pair-wise comparisons indicated 
TABLE 1

Speech performance: Mean performance across speech indices for each age (SD)

\begin{tabular}{|c|c|c|c|c|c|}
\hline Measures & 4 years & 5 years & 6 years & $F$ value & $\begin{array}{c}\text { Multiple } \\
\text { comparison }^{a}\end{array}$ \\
\hline $\begin{array}{l}\text { Total speaking } \\
\text { time (second) }\end{array}$ & $16.45(12.97)$ & $29.30(9.44)$ & $57.00(42.28)$ & $12.87^{* *}$ & $6>5^{*}, 6>4^{* *}, 5>4^{*}$ \\
\hline Total morphemes & $28.07(20.05)$ & $40.60(14.84)$ & $73.00(48.69)$ & $9.69^{* * *}$ & $6>4 * *$ \\
\hline $\begin{array}{l}\text { Total pausing } \\
\text { time (second) }\end{array}$ & $6.25(6.57)$ & $10.80(6.62)$ & 18.67 (14.73) & $7.04 * *$ & $6>4^{*}$ \\
\hline $\begin{array}{l}\text { Number of } \\
\text { motion verb }\end{array}$ & $4.47(2.48)$ & $7.33(2.61)$ & $10.07(5.55)$ & $9.39 * *$ & $6>4^{* *}, 5>4^{*}$ \\
\hline $\begin{array}{l}\text { Number of } \\
\text { left/right term }\end{array}$ & $0.00(0.00)$ & $0.40(0.74)$ & $2.20(3.01)$ & $7.2^{*}$ & $6>4^{*}$ \\
\hline $\begin{array}{l}\text { Number of } \\
\text { landmark }\end{array}$ & $0.93(1.22)$ & $1.67(1.23)$ & $4.73(4.62)$ & $9.48^{* *}$ & $6>4 * *$ \\
\hline
\end{tabular}

*** $p<.001, * * p<.01, * p<.05$. ${ }^{\text {a }}$ Number indicates age of children.

that the number of children who mentioned left/right terms and landmarks were much lower for 4-year-olds than for 6-year-olds (two-tailed McNemar test, $p=.0156$ and .0156 , respectively).

When individual patterns of change with age were examined, no children were found to have decreased their use of left/right terms with age. However, three children decreased their use of landmarks from 4 to 5 years of age, and two other children showed a similar decrease from 5 to 6 years of age. All remaining children either increased or maintained the same number of references to landmarks. Overall, speech performance was generally found to increase with age.

\section{Gestural performances}

A repeated-measures ANOVA was conducted on the total number of gestures produced and the frequency of gestures per second. A main effect of age was found for the total number of gestures, $F(2,28)=6.80, p<.01$, and for gesture frequency, $F(2,28)=3.38, p<.05$. Post hoc comparisons (Bonferroni, $p<.05)$ indicated that 5-year-olds $(M=10.13, S D=3.94)$ and 6-year-olds $(M=13.80, S D=9.57)$ produced more total gestures than 4-year-olds $(M=6.40, S D=3.99)$. In addition, 4-year-olds $(M=0.44$, $S D=0.27)$ produced significantly more gestures per second than 6-yearolds $(M=0.27, S D=0.16)$, but did not different significantly from 5-yearolds $(M=0.38, S D=0.18)$. It is clear that although the total production of gestures increased with age, the frequency of gesture use decreased with age. Because as children get older the total speaking time increases even though they can produce many gestures, the frequency of gesture decreased with age. 


\section{Direction of gestures}

Central to the study, I next examined how children's gestures indicate the underlying frame of reference. As described above, children were categorised by whether the direction of their gestures consistently matched the target location. The number of children meeting each directional category is shown in Figure 2. A chi-square analysis revealed a significant difference between age and number of children in each category, $\chi^{2}(4, n=14)=25.4, p<.001$. A residual analysis indicated that there were significantly more 4 -year-olds in the match group, whereas significantly more 5 -year-olds were in the mixed group, and finally, more 6-year-olds were in the mismatch group. In order to test if children changed the direction of their gestures over the 3-year period, Cochran's $Q$ test was carried out for each category, with McNemar tests for post hoc pair-wise comparisons. It revealed that there was a significant difference in the number of children who were categorised in the match group, $Q(2,14)=14.250, p<.001$, in the mismatch group, $Q(2,14)=6.000$, $p<.05$, and in the mixed group, $Q(2,14)=7.800, p<.05$. Pairwise comparisons indicated that the number of 4-year-olds who were categorised as the match group was significantly more than 5- and 6-year-olds (twotailed McNemar test, $p=.008$ and .016, respectively). As for the number of children who were categorised in the mismatch group, there were marginally significant differences between 6-year-olds and 4- and 5-year-olds. Six-yearolds were more often categorised as the mismatch group compared with

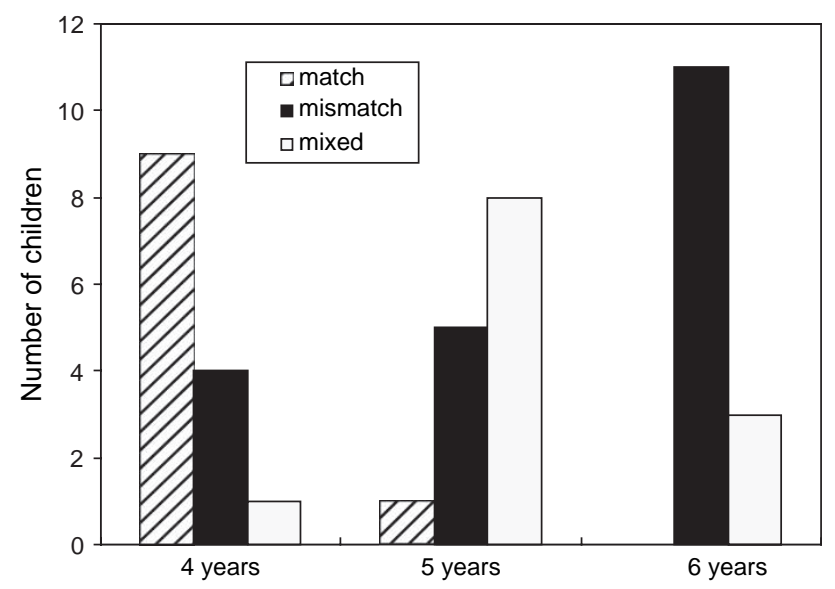

Figure 2. The number of children who are categorised in the match group, mismatched group, and mixed group at each age. Children were categorised as match group if they consistently produced gestures that fell within \pm 45 degrees of the target vector. In contrast, when children consistently produced gestures that did not match with their actual route, they were categorised within the mismatch group. Children who used both match and mismatch gestures in their descriptions were categorised as the mixed group. 
4- and 5-year-olds (two-tailed McNemar test, $p=.070$ and .070 , respectively). There were significantly more 5 -year-olds who were categorised as the mixed group compared to 4-year-olds (two-tailed McNemar test, $p=.016$ ).

These results indicate that younger children tend to indicate their actual route quite directly, though this same tendency does not exist in older children.

As for individual patterns from 4 to 6 years of age, although six children changed from match to mismatch and three children changed from match to mixed, none of the children changed from mismatch to match.

\section{The use of gesture space}

After an angular transformation, a repeated-measures ANOVA was conducted to test if the proportion of gesture produced in each gesture space differed as a function of age. The result indicated that there was a main effect of age on the use of centre space, $F(2,26)=8.23, p<.01$. A post hoc comparison (Bonferroni, $p<.05$ ) showed that centre space was used by 6 -year-olds $34 \%$ of the time, which was significantly greater than 4-year-olds at $7 \%$, but not significantly different from 5-year-olds, who showed $20 \%$ usage.

As shown in Table 2, more than half of gestures in any age group were produced in the periphery, and the use of centre-centre space accounted for less than $10 \%$ of gestures. Furthermore, roughly half of all gestures were produced outside of the front space in early childhood, and with increasing age, the production of gestures in the extreme periphery decreased, while the production of gestures in centre space increased. That is, the space used for gestures gradually reduced in size with increasing age.

\section{Gesture morphology}

After an angular transformation, a repeated-measures ANOVA was carried out separately for the proportion of gestures involving a hand raised above the shoulder or an elbow extension. A main effect of age was seen on

TABLE 2

The proportion of use of gesture space $(S D)$

\begin{tabular}{lccclc}
\hline & 4 years & 5 years & 6 years & F value & Multiple comparison $^{a}$ \\
\hline Centre-Centre & $0.04(0.08)$ & $0.06(0.14)$ & $0.07(0.09)$ & 0.3 & \\
Centre & $0.07(0.12)$ & $0.20(0.19)$ & $0.34(0.22)$ & $8.23^{* *}$ & $6>4^{* *}$ \\
Periphery & $0.64(0.36)$ & $0.52(0.27)$ & $0.50(0.20)$ & 2.09 & \\
Extreme periphery & $0.25(0.35)$ & $0.22(0.30)$ & $0.09(0.15)$ & 1.68 & \\
& & & &
\end{tabular}

\footnotetext{
$* * p<.01 .{ }^{\mathrm{a}}$ Number indicates age of children.
} 
the proportion of gestures with a hand raised above the shoulder, $F(2,26)=$ $3.39, p<.05$, and on the proportion of gestures involving a fully extended elbow, $F(2,26)=5.18, p<.05$. Post hoc comparisons (Bonferroni, $p<.05$ ) showed that the proportion of gestures with a hand raised above the shoulder was significantly greater for 4 -year-olds $(55 \%)$ than for 6-year-olds $(26 \%)$, but not significantly greater than that for 5 -year-olds $(52 \%)$. In addition, 4- $(48 \%)$ and 5 -year-olds $(43 \%)$ produced a greater proportion of gestures with extended elbows than did 6-year-olds $(8 \%)$.

\section{Number of gesture units and gesturing time}

I next examined whether the duration and the proportion of time spent gesturing decreased with age by calculating the number of gesture units and gesturing time. A repeated-measures ANOVA revealed a main effect of age on gesture units, $F(2,26)=9.26, p<.01$. Post hoc comparisons (Bonferroni, $p<.05)$ showed that 6 -year-olds $(M=5.64, S D=5.26)$ produced more gesture units than either 4-year-olds $(M=1.43, S D=0.65)$ or 5-year-olds $(M=2.29, S D=2.40)$. Examination of data from individual children showed that there were three children who showed a decrease in the number of gesture units from 4 to 5 years of age, but none of the children showed a decrease from 5 to 6 years of age.

Total gesturing time (in seconds) was calculated by adding the time spent producing gesture units during a description. A repeated-measures ANOVA on total gesturing time showed a main effect of age, $F(2,26)=4.76, p<.05$. Post hoc comparisons (Bonferroni, $p<.05)$ showed that 6-year-olds $(M=$ $26.40, S D=16.06)$ produced longer gesture times than 4-year-olds $(M=$ $13.44, S D=9.94)$, but the difference between the 6-year-olds and the 5-yearolds $(M=21.99, S D=7.77)$ was not significant.

The proportion of gesturing time in a description was calculated by dividing the total gesturing time by the total speaking time. A repeatedmeasures ANOVA demonstrated a main effect of age on the proportion of gesturing time, $F(2,26)=5.09, p<.05$. Although 4-year-olds $(M=0.87$, $S D=0.40$ ) showed the greatest proportion of gesturing time, post hoc comparisons (Bonferroni, $p<.05$ ) indicated they did not differ significantly from either 5 - $(M=0.80, S D=0.21)$ or 6 -year-olds $(M=0.54, S D=0.30)$, though these latter two age groups did differ significantly. Thus, the number of gesture units and total gesturing time increased with age. But the proportion of gestures in descriptions decreased with age, because of a substantial increase in the speaking time.

\section{Relation between speech and gesture indices}

Finally, a correlation analysis was conducted to assess the relationship between the use of verbal descriptors (e.g., left/right terms and landmarks) 
TABLE 3

A correlation analysis between gestural and speech indices $(n=42)$

\begin{tabular}{lccccc}
\hline & $\begin{array}{c}\text { Total } \\
\text { number of } \\
\text { gestures }\end{array}$ & $\begin{array}{c}\text { Frequency of } \\
\text { gestures }\end{array}$ & $\begin{array}{c}\text { Number } \\
\text { of gesture } \\
\text { unit }\end{array}$ & $\begin{array}{c}\text { Proportion } \\
\text { of elbow } \\
\text { extension }\end{array}$ & $\begin{array}{c}\text { Proportion of } \\
\text { hand raised } \\
\text { above the } \\
\text { shoulder }\end{array}$ \\
\hline Number of landmark & $.60^{* * *}$ & $-.38^{*}$ & $.72^{* * *}$ & -.24 & $-.38^{* *}$ \\
Number of left/right term & $.34^{*}$ & -.28 & $.44^{* * *}$ & $-.31^{*}$ & -.27 \\
\hline
\end{tabular}

*** $p<.001, * * p<.01, * p<.05$.

and gesture indices (Table 3). All three of the age group was collapsed for this analysis because the 4- and 5-year-olds had used much fewer left/right terms and landmarks than the 6-year-olds. The gesture indices used were the total number of gestures, gesture frequency, the number of gesture units, and the proportion of gestures involving the hand raised above the shoulder or an elbow extension.

As Table 3 shows, the number of landmarks described was positively and significantly correlated with total number of gestures and the number of gesture units, but negatively correlated with gesture frequency and the proportion of gestures with a hand raised above the shoulder. Similarly, the number of left/right terms used was positively correlated with total number of gestures and the number of gesture units, but negatively correlated with the proportion of gestures involving an elbow extension. These relationships demonstrate that as children develop their ability to verbally indicate landmarks and/or state left/right directionally, their total number of gestures and the number of gesture units also increase. In contrast, using descriptions of landmarks was related to a smaller proportion of gestures raised above the shoulder, and verbally indicating left/right related to a smaller proportion of gestures with the elbow extended fully.

\section{DISCUSSION}

The present study longitudinally investigated developmental changes in the use of frames of reference in 4- to 6-year-olds by examining the spontaneously produced gestures and speech used when describing their route home from nursery school. As age increased, an increase was seen in the number of references made to turns on the route with gestures and speech, the total speaking time, total morphemes pauses, left/right usage, references to landmarks, and the total number of gestures; there were especially striking differences between the responses of 4-year-olds compared with their responses 2 years later, at age 6 . 
Based on these results I will discuss (1) the change in frame of reference usage during preschool age, as inferred from the results analysing speech and gestures, and (2) the theoretical implications of inferring frame of reference from gestures.

\section{Frame of reference: Developmental changes}

When the children studied were 4 years old, they described fewer landmarks, used no left/right terms in their descriptions, and the proportion of the time spent gesturing in their descriptions was greater than when they were 6. As for gestural characteristics, 4-year-olds tended to produce more gestures that consistently matched the actual direction they were referring to, and their gesture space and gesture morphology were larger than when they were older. These tendencies are partially consistent with the gestural characteristics found in linguistic communities that primarily rely on an absolute frame of reference. However, because it is difficult to fully understand the cardinal direction for the children in the study, it is not clear whether they were able to use space absolutely, as in Levinson's description (2003). Nevertheless, most of the 4-year-olds appeared to recall the image of their route by orienting the gestures accompanying their speech from a basic origo (the location of the gesturer), consistent with the geoanchored gestures proposed by Kita (1998). The fewer gesture units produced by the younger children further suggest that their mental image of the route was relatively continuous; that is, it was not segmented into a series of turns. Taken together these results regarding gestures and speech suggest that 4-year-olds mainly use an actual environmental frame of reference in that their body seems to be closely connected to the physical environment surrounding them.

In contrast, when the children were 6 years old, most of them could explain their route using left/right terms and landmarks, rather than pointing directly to the environment. Furthermore, it was found that gesture morphology and gesture space were smaller when they were 6 as compared to when they were 4 ; with increasing age, the older children appeared to create a virtual space in front of themselves. Thus, the older children appeared to explain their route based on their body as transposed origo in that they attempted to mentally jump to various points along the route without gestural orientation, and they could use their own body as a reference point to indicate left or right. From this, it can be inferred that 6-year-olds mainly use an abstract environmental frame of reference in that their body appears to be detached from the actual environment and they rely on their mental image of particular points along the route. Furthermore, the finding that the children produced more gesture units when they were 6 than 
in the preceding 2 years suggests that their spatial representation became increasingly segmented with age.

In moving from an actual environmental frame of reference at age 4 to an abstract environmental frame of reference at age 6, the 5-year-olds fell inbetween. When the children were 5 , they produced fewer verbal descriptions and gestures than when they were 6 , but the characteristics of their gestures were similar to those produced as 6-year-olds; some of the 5-year-olds produced gestures without direct orientation to the actual route. When the 5 -year-olds described their route nearby the nursery school, most of them produced gestures that directly indicated the actual direction, but as their reference location moved away from the nursery school, their gestures did not tend to match the actual locations they were describing. Taken together, age 5 appears to be a transitional age in which children move away from using an actual environmental frame of reference and incorporate more abstract environmental responses.

There are two main explanations for this developmental shift in frame of reference from actual to abstract environment. First, the development of linguistic encoding skills can be considered a factor that influences this shift. With linguistic encoding skills, children would be able to recall the route based on landmarks in order, without gesturally indicating the actual direction. The finding that 6-year-olds who mention numerous landmarks can describe their route without gestural orientation supports this idea. The relationship between the verbalisation of landmarks and left/right terms, and increasingly segmented gesture units and smaller gesture morphology is also consistent with this. In contrast, when the children in the present study were younger, they may have adopted direct and continuous expressions of the actual route to compensate for their more limited ability to encode landmarks or directionality. Kita (2003) argued that turning the gaze or hands into the visible part of the environment seems to be helpful in inferring the invisible environment. The gestures produced by the 4 -year-olds in the present study may have helped them to recall and describe the route. However, even though the 6-year-olds could use verbal descriptors of left/ right and landmarks to indicate their route, they continued to gesture. Gestures may serve not only to communicate information, but also to recall a particular landscape in the middle of a route or directionality.

A second explanation for the developmental shift found in the present study, is that commuting experiences cause a change in frame of reference. That is, accumulating the commuting experiences would result in a learning effect. As children get older, they can better understand the positional relationship between the nursery school and neighbouring landmarks. With this knowledge, older children could describe their route without relying on the actual environment. In the present study, when the children were 6 years old, they were also asked to describe the route to the nearest library, located 
$700 \mathrm{~m}$ away from the nursery school. Even though all of the children had gone to the library together approximately seven times before, most children answered 'I do not know' or 'I forgot'. Given the discrepancy between the ability of the 6-year-olds to describe their familiar route home as compared with the relatively unfamiliar route to the library, it can be postulated that the change in frame of reference seen between 4 and 6 years of age resulted from increased familiarity and practice following their route home.

These two explanations (the development of linguistic encoding skills and experience with a route) complement each other. As children commute between their nursery school and home nearly every day, they gradually become familiar with the environment and encode landmarks along the route. Indeed, these interactions between commuting experience and language development may well prescribe the frame of reference used.

\section{Theoretical implication}

The present study demonstrated that the frame of reference used by children during their preschool years to describe a familiar route shifts from an actual environmental frame of reference, directly relying on the external environment, to an abstract environmental frame of reference that is based on encoded landmarks. Next, it may be argued that certain theoretical implications can be drawn from this developmental change in frame of reference.

Piaget et al. (1960) argued that there is a shift from an egocentric to an allocentric frame of reference with age, and that children predominantly use an egocentric frame of reference until the age of 7 . Children using an egocentric frame of reference recall routes based on their own body movement, similar to the movements made during their actual travel, rather than relying on the external environment. However, in the present study, as 4-year-olds, the children tended to use gestures to directly indicate the external environment, but around age 6, they were able to describe their route with gestures confined to a smaller space by referring to landmarks. Although the present data partially support Piaget's finding that children develop the ability to refer to landmarks with age, the data go beyond this to illuminate the relationship between children and their surrounding environment that was obscure in Piaget's study. The present study demonstrated that young children strongly rely on and connect with the actual space surrounding them as if they are embedded within the environment. As they get older, they can disconnect themselves from the environment, and use a limited space in front of themselves symbolically to create gestures that are not directly oriented to the physical environment. This tendency supported findings of the previous cross-sectional study on route descriptions of preschool children (Sekine, 2006). This could exclude, although not 
conclusively, the possibility that changes in the frame of reference observed in the present study was caused by the training effect of repeating route descriptions on three occasions.

Moreover, the result of the present study is consistent with previous studies examining small-scale environments (Craton et al., 1990; Newcombe \& Huttenlocher, 1992). For example, this developmental change appears compatible with the classification of use of space suggested by Presson and Somerville (1985) into primary and secondary uses. They defined primary use as involving practical orientation to the immediate perceived surroundings and secondary use as dealing with symbolic spatial information to which one is not directly oriented. Within this framework, the results of the present study indicate that 4-year-olds tend to use mainly primary actual space, whereas 6-year-olds can use secondary symbolic space, and 5 -year-olds fall into a transitional stage. Consistent with this, Bluestein and Acredolo (1979) reported that when children are asked to use a map to find a hidden target in an environment, children over 5 years of age could typically find the target, even though the direction of the map did not match the actual environment.

However, as Presson and Somerville (1985) have implied, one may argue that a frame of reference that is inferred from gestures and speech in communication does not necessarily reflect a frame of reference that is used to mentally encode. This study can not rule out such a possibility. But Majid, Bowerman, Kita, Haun, and Levinson (2004), who reviewed recent researches on frames of reference used in linguistic and non-linguistic expressions, including gesture, reported that in certain spatial phenomena such as the directionality of a moving object and the change of a human location, frames of reference that are revealed in communicative gestures and those that are revealed in non-communicative tasks, correspond to each other very closely. These findings support the interpretation of the present study that the changes in the frame of reference reflected in gestural expressions, to some extent reflect changes in mental coding. However, further investigations are needed to clarify how the frame of reference used for communicating is related to those used for mental coding.

In addition, how the use of frames of reference change after the preschool age remains to be determined. The use of an actual environmental, an abstract environmental, or a combination of these frames of reference is often observed in school-aged children and adults. In addition to linguistic ability and experience with a route, many other factors, including mapreading skill and considering the listener's knowledge level, can influence the use of a particular frame of reference. Further investigations are required to determine how these factors prescribe changes in frame of reference.

In summary, by focusing on gestures produced spontaneously during route descriptions, the present study demonstrated that gestures and speech 
develop in parallel above 2 years of age. It was also found that 4-year-olds tend to orient themselves to the physical environment surrounding them and to produce gestures that directly and continuously indicated their actual route in a large gesture space, suggesting the use of an actual environmental frame of reference. By age 6, children can segment and use space symbolically in both their gestures and their speech, and they can use fixed external landmarks even in the period when Piaget et al. (1960) assumed that children predominantly use egocentric frame of reference. These results imply that younger children's route representations are more continuous and anchored to the actual environment than those of older children. In contrast, older children's representations seem to be more segmented and anchored to their mental image of particular points along the route than those of younger children. In addition, the present study suggests that spontaneous gestures can provide a useful index by which to measure and understand children's spatial knowledge, and unlike conventional indices this measure does not cue participants to use a particular frame of reference.

\section{REFERENCES}

Blades, M., \& Medlicott, L. (1992). Developmental differences in the ability to give route directions from a map. Journal of Environmental Psychology, 12, 175-185.

Bluestein, N., \& Acredolo, L. (1979). Developmental changes in map-reading skill. Child Development, 50, 691-697.

Craton, L. G., Elicker, J., Plumert, J. M., \& Pick, H. L. (1990). Children's use of frames of reference in communication of spatial location. Child Development, 61, 1528-1543.

Doherty-Sneddon, G., \& Kent, G. (1996). Visual signals and the communication abilities of children. Journal of Child Psychology and Psychiatry, 37(8), 949-959.

Gauvain, M., \& Rogoff, B. (1989). Ways of speaking about space: The development of children's skill in communicating spatial knowledge. Cognitive Development, 4, 295-307.

Hart, R. A., \& Moore, G. T. (1973). The development of spatial cognition: A review. In P. M. Downs. \& D. Stea (Eds.), Image and environment (pp. 246-288). Chicago: Aldine Publishing.

Haviland, J. B. (1993). Anchoring, iconicity, and orientation in Guugu Yimithirr pointing gestures. Journal of Linguistic Anthropology, 3(1), 3-45.

Iverson, J. M. (1999). How to get to the cafeteria: Gesture and speech in blind and sighted children's spatial descriptions. Developmental Psychology, 35(4), 1132-1142.

Kita, S. (1998). Expressing turns at an invisible location in route direction: The interplay of speech and body movement. In E. W. B. Hess-Luttich, J. E. Muller, \& A. V. Zoet (Eds.), Sign and space: Raum und zeichen (pp. 160-172). Tübingen, Germany: Gunter Narr.

Kita, S. (Ed.).(2003). Interplay of gaze, hand, torso orientation, and language in pointing. Pointing: Where language, culture, and cognition meet (pp. 307-328). Mahwah, NJ: Lawrence Erlbaum Associates.

Kendon, A. (1980). Gesticulation and speech: Two aspects of the process of utterance. In M. R. Key (Ed.), The relation between verbal and nonverbal communication (pp. 207-227). The Hague: Mouton. 
Levinson, S. C. (2003). Space in language and cognition. Cambridge, UK: Cambridge University Press.

Majid, A., Bowerman, M., Kita, S., Haun, D. B. M., \& Levinson, S. C. (2004). Can language restructure cognition? The case of space. TRENDS in Cognitive Sciences, 8(3), 108-114.

McNeill, D. (1992). Hand and mind. Chicago: University of Chicago Press.

McNeill, D. (2005). Gesture and thought. Chicago: University of Chicago Press.

Newcombe, N., \& Huttenlocher, J. (1992). Children's early ability to solve perspective-taking problem. Developmental Psychology, 28(4), 635-643.

Piaget, J., \& Inhelder, B. (1956). The child's conception of space. London: Routledge \& Kegan Paul.

Piaget, J., Inhelder, B., \& Szeminska, A. (1960). The child's conception of geometry. London: Routledge and Kagan Paul.

Presson, C. C. (1980). Spatial egocentrism and the effect of an alternate frame of reference. Journal of Experimental Child Psychology, 29, 391-402.

Presson, C. C., \& Somerville, S. C. (1985). Beyond egocentrism: A new look at the beginnings of spatial representation. In H. M. Wellman (Ed.), Children's searching: The development of search skill and spatial representation (pp. 1-26). Hillsdale, NJ: Lawrence Erlbaum Associates.

Rauscher, F. H., Krauss, R. M., \& Chen, Y. (1996). Gesture, speech, and lexical access: The role of lexical movements in speech production. Psychological Science, 7(4), 226-231.

Sekine, K. (2006). Youji ni okeru kuukansanshouwaku no hattatu: Keirosetsumei ni okeru kotoba to miburi ni yoru kentou. [Developmental changes in spatial frame of reference among preschoolers: Spontaneous gestures and speech in route descriptions]. The Japanese Journal of Developmental Psychology, 17(3), 263-271.

Waller, G. (1986). The development of route knowledge: multiple dimensions? Journal of Environmental Psychology, 6, 109-119. 